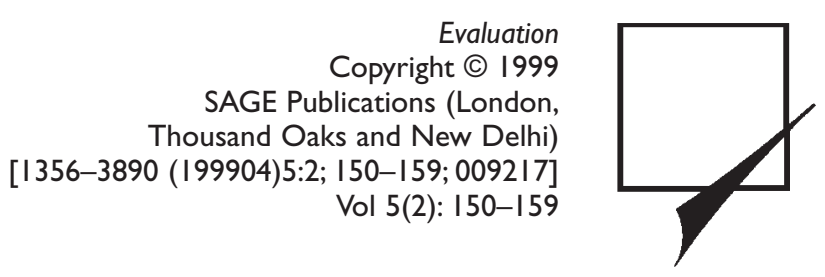

\title{
Evaluation and Performance Management in Government
}

\author{
IAN C. DAVIES \\ European Court of Auditors
}

This article discusses the link between evaluation and performance management, drawing the audience's attention to how they are different and how they are complementary (e.g. where evaluation provides the framework to develop 'good' indicators, where performance data can be used for evaluation and an interdependent relationship). Having looked at some of the problems and pitfalls, the article concludes by focusing on this complementarity and argues that evaluators can and should contribute to debates about improving performance within organizations.

\section{Introduction}

That governments should apply themselves to continuously improving their performance, particularly with respect to the management of public funds and the stewardship of public assets, appears now to be a generally accepted principle of good governance.

In the European context, this principle underlies the administrative reform efforts of a good number of governments, and is reflected in the widespread expectation that policy and resource allocation decisions be based on sound information about the performance of public programmes.

From the perspective of the European Union's external auditor, the European Court of Auditors, the importance of sound financial management of public funds is such that the Court is expanding its performance audit practice. Being closely associated with this effort, issues related to government performance, its monitoring, evaluation and reporting are ever present in my work.

Drawing on my current practice, and experience working with a number of governments in Western democracies, my presentation will contrast the uses of evaluation and performance management in government settings and look at what evaluation knowledge can contribute to administrative reform efforts.

Now performance management may be construed generally as the process by which we collect performance information and use it for improvement, by drawing on a range of evaluative processes such as performance measurement and evaluation. From this vantage point, it would appear logical to contrast 
performance measurement and evaluation within the performance management approach.

However, for the purpose of our discussion today, I will use the term performance management to mean performance measurement (as different from evaluation) and the use of performance monitoring information by governments to re-orient administrative culture away from a procedural one to a culture that values the achievement of results.

\section{What is Performance Management?}

Performance management can be thought of as a set of systematic efforts, initiatives and processes that have a number of characteristics. They attempt to:

- Define performance in terms of results (outputs, outcomes, effects, impacts, etc.);

- Set measurable levels of intended achievement (performance targets, service standards, etc.);

- Determine the extent to which results are achieved using performance indicators (performance measurement, performance monitoring, etc.);

- Provide an accounting for the achievement of results in light of the resources utilized (performance reporting, effectiveness reporting, value-for-money accounting, etc.);

- Base resource allocation decisions on performance information (performance budgeting, results-based budgeting, etc.).

This pattern of identifying intended results, measuring, reporting and using the information for decision-making typifies the way many jurisdictions define performance management. Here are some examples to illustrate my point:

Performance management is a process of defining a mission and desired outcomes, setting performance standards, linking budget to performance, reporting results, and holding public officials accountable for those results. (The National Academy of Public Administration, cited in English and Linquist, 1998)

Performance measurement is the ongoing monitoring and reporting of programme accomplishments, particularly progress towards pre-established goals. (US General Accounting Office, 1998)

Performance management is managing and reporting based on what programmes are achieving for citizens and at what cost. This implies agreeing on expected outcomes, measuring progress toward them and using that information to improve performance and report results. (The Office of the Auditor General of Canada, cited in English and Linquist, 1998)

Performance management is using performance information effectively and performance measurement is obtaining the requisite information. (KPMG, cited in English and Linquist, 1998)

\section{The Link between Evaluation and Performance Management}

Evaluation and performance management can be thought of as different from each other yet complementary. 


\section{Evaluation 5(2)}

They are usually differentiated in two ways: first, by the frequency of activity, i.e. evaluation is a one-off exercise whereas performance management is continuous; and second, by the nature of the questions each approach addresses, i.e. evaluation asks the 'why and how' questions, whereas performance management asks the 'what' questions.

The European Commission (1997), for example, characterizes the difference between the two as follows:

... evaluation must be distinguished from monitoring.... It is a continual process, carried out during the execution of the programme, with the intention of immediately correcting any deviation from operational objectives. Evaluation, on the other hand, is specifically conducted at a discrete point in time in the life cycle of a programme, and consists of an in-depth study.

With respect to the nature of the questions, Joe Wholey (United States General Accounting Office, 1998) states that evaluation is usually about individual systematic studies to assess how well a programme is working, whereas performance measurement is typically concerned with the ongoing monitoring and reporting of accomplishments.

Evaluation and performance management are seen as complementary, and here there is a 'range of complementarity': some jurisdictions see them as having a mutually beneficial relationship, so that evaluation provides the framework to develop 'good' indicators for performance monitoring purposes, and performance data that are collected in an ongoing manner can be used for evaluation purposes.

As well, there is the view that evaluation and performance management are interdependent, that is, the successful existence of one depends on the effective realization of the other.

Burt Perrin (1999), for example, tells us how performance measurement ‘. . needs to be complemented with more robust and comprehensive evaluation approaches in order to assess the nature of programme impact, to get at causality, and to explore the potential of alternative approaches'.

The European Commission (1997) gives us evaluation dependent on performance measurement: 'Monitoring is of key importance to improving programme performance, and successful evaluation hinges upon successful monitoring, for example because monitoring often generates data which can be used in evaluation'.

\section{The State of Evaluation and Performance Management in Govern- ment}

For all the statements about the desirability of complementarity or the necessity of interdependence, a balanced mix of evaluation and performance management in government is not common.

In my experience, assessment approaches tend to cluster mostly at one or the other end of the 'evaluative continuum'. Governments tend to favour more evaluation or performance management, although there appears to be a discernible 'migration to the middle' as the benefits of complementarity or interdependence become apparent.

Some governments do, however, attempt to use a mix of approaches. For 
example, Mexico's Public Administration Modernization Programme (PAMP) includes the establishment of a 'performance and service to customer' culture involving extensive programme follow-ups and evaluations, specified quality service goals and measures, and a systematic evaluation of results based on public servant performance.

The government of Canada uses three different tools to assess its programmes: evaluation, review and audit. The President of the Treasury Board issues an annual report to Parliament (Treasury Board of Canada, 1998) on the state of evaluative efforts in the government and provides internet access to departmental performance reports and evaluations.

Overall however, performance management seems to be fairly pervasive, and, to my mind, is a little more common these days in government than is evaluation. For example, in 1995, the government of Iceland implemented a formal policy strategy for modernizing public administration based on 'simplification, responsibility and results'. The current reporting system of activity indicators invites all government agencies and institutions to submit individual business plans and performance goals.

In another part of the world, the South African government's Reconstruction and Development Programme (RDP) has as its guiding principle quality service to the people through transformation of public service delivery. The initiative, born in the White Paper on the Transformation of the Public Service, consists essentially of eight broad commitments known as the Batho Pele principles which include: citizen consultations, service standards, service access, courtesy, service information, openness and transparency, citizen redress and value for money.

\section{Understanding the Ascendancy of Performance Management}

What factors might explain the apparent predominance of performance management and, in a lot of cases, the migration of government assessment practices from evaluation towards performance management?

One of the main reasons for this state of affairs lies in the progressive disuse of evaluations by governments because they are not seen to meet the expectations of decision-makers who hold the view that: evaluations are costly, take time and are difficult to justify in tight fiscal situations; evaluation reports are long and complex with few people reading or using them; evaluations are more often formative than summative so that issues of programme relevance are seldom addressed; and the information provided by evaluation suffers from lack of timeliness and is seldom considered useful for resource allocation or budgeting decisions.

Another reason is the fertile ground provided by neo-conservative policies for the rise of managerialism and the development of what has been dubbed 'the new public management' in government. The 'business knows best' approach to managing public programmes values the use of techniques such as strategic and operational planning, a focus on 'deliverables' and results, results-based incentives and accountability, the citizen as customer, service standards, etc. There is a natural compatibility between this corporatist approach to government administration and the world of performance, reflected in part by common usage 


\section{Evaluation 5(2)}

of terms such as performance indicators, performance-related pay, benchmarking, quality service standards, balanced scorecard, etc.

Lastly, an important factor affecting the development of performance management in government is the growing influence of the accounting and audit community. This has been occurring progressively over the last 20 years or so as the accounting and auditing professions turn their attention to non-financial areas of public administration. Most notable is the development of non-financial audit approaches by external legislative auditors, e.g. comprehensive audit, value-formoney audit, performance audit, environmental audit.

The reinforcement, by the accounting and audit community, of the need for regular, i.e. annual, accountability reporting with a focus on results achieved, related costs, performance indicators, etc., is consistent with the performance management paradigm. This influence has, among other things, driven the development of internal control and internal audit functions in government with an emphasis on management control frameworks, results-based budgeting, performance reporting, etc.

A good indication of the extent to which performance management, and the use of performance indicators and performance measures, is increasingly rooted in the administration of governments, is the evolving role of the external/legislative auditor. For example, Western Australia, New Zealand, Sweden, the US, England and Wales now carry out audits of performance indicators and issue opinions on the indicators and the associated information.

In the process, auditors appear to be gaining a 'market advantage' within government over evaluators, not so much for methodological reasons, but because of the congruity of that professional culture with the values that underpin current administrative reforms and the perceived legitimacy conferred by legislated mandates, well-established professional bodies and legally sanctioned accreditation.

\section{The Pitfalls of Performance Management}

The problems with performance management in the public sector continue to be well documented by the evaluation community, at times with zealous glee. Allow me to quickly summarize what we know.

A first and very real risk is the misuse of performance information, which happens in a myriad of ways, including the following:

- Inadequate indicators that do not measure what they are intended to, i.e. what evaluators would call 'poor construct validity'. This situation often arises when a performance assessment process is implemented hurriedly, under pressure, and indicators are chosen in haste or without sufficient consideration;

- Use of performance monitoring information in a manner that assumes causality in relation to outcomes. This 'classic' pitfall is due typically to a lack of appropriate knowledge and know-how (often due to insufficient resources to obtain the necessary expertise); as well, the link between activities and outcomes is usually taken for granted by those who carry out the programme and is treated as something 'obvious'; 
- Use of performance information to ends for which it was not intended. Information is 'hijacked' by senior management or political masters who interpret it in their own ways to suit other purposes, and/or the information is communicated without sufficient explanation or context, e.g. to fit the requirements of media 'sound bites'.

Another important risk, and one that in my mind constitutes the Achilles' heel of performance management, is goal displacement. This phenomenon occurs when performance management creates incentives that direct effort towards meeting the requirements of measuring and reporting to the detriment of the programme's relevance; in short, when performance indicators and targets overtake the programme's raison d'etre.

The risk of falling into that trap increases under the following conditions: insufficient attention is given to the implementation process; the measurement approach reduces dynamic and complex multi-dimensional programmes to simplistic formulae and/or mechanistic linear processes; the focus of the assessment effort is on cosmetics rather than fundamentals; and, likely the surest way of producing goal displacement, there is failure to involve stakeholders and obtain buy-in.

The problems associated with goal displacement can be exacerbated by what could best be called 'perverse incentives'. For example, raising the stakes associated with performance management increases the likelihood that people will 'work first to the indicators' and produce by whatever means the required performance information. This, in turn, increases the probability of inaccurate data and unreliable information.

Another set of problems arises from the limitations inherent to measurement. As the proponents of performance management like to remind us, 'what gets measured gets done'; however, the consequence of that credo is that what is difficult to measure could likely fall by the wayside, especially under 'high stakes' conditions.

Low-probability programme technologies, i.e. 'soft' programmes such as social interventions, are particularly vulnerable to lack of attention to what is really important because it is difficult to measure (often meaning 'quantify'). The risk is all the more significant that, typically, most government interventions fall into this category. As Burt Perrin states in 'Effective Use and Misuse of Performance Measurement': 'Many activities in the public policy realm, by their very nature, are complex and intangible and cannot be reduced to a numerical figure.... What is measured, or even measurable, often bears little resemblance to what is relevant' (Perrin, 1999).

In general, top-down implementation of performance management processes, that are not supported by genuine stakeholder buy-in, and that set up high stakes/high risk consequences for staff, are highly likely to be detrimental to relevant performance.

\section{The Contribution of Evaluation}

The main pitfalls in performance management can be sorted into two categories: the 'technical' difficulties such as problems of internal validity, construct validity, 
instrumentation, etc., and the 'people issues' such as trust, buy-in, organizational politics, etc.

In my experience, the most common suggestions with respect to what evaluation can contribute to improve performance management appear to address primarily the technical difficulties.

This is especially true with problems of outcome attribution, given that this an area in which evaluation can rightfully lay claim to substantive know-how. The extent to which an outcome and its observed variance can be attributed to the programme remains a key methodological hurdle in goal-directed evaluation work.

As a result, evaluation can contribute significantly to resolving attribution questions by, among other things, providing know-how for modelling causality assumptions. Evaluation has developed ways of representing the cause and effect linkages of a programme, using, among other approaches, '... an open systems approach, [as a] framework for conceptualizing interactions between the programme and its environment' (McDavid, 1998). Indeed, the logic model is one of the basic tools of evaluation for working with results-based assessments.

It is unrealistic, however, to think that logic modelling will, in itself, resolve the methodological difficulties of outcome attribution, especially in the usual performance management implementation context.

Typically, the practised evaluator will attempt to find practical solutions that, within the constraints of methodology and resources, can support a credible assessment. For example: sorting out the 'political' goals from the more practical outcomes; involving stakeholders so they can 'validate' the performance measures; pre-testing performance indicators; using multiple indicators which are reviewed, updated and changed regularly; using data collection methods which are independent of one another; getting an independent assessment of the performance management process, e.g. through peer review.

On the other hand, the 'people' issues, which in my experience are seriously neglected in performance management initiatives, are not considered sufficiently as an area in which evaluation can make a significant contribution.

Yet the 'people' issues are critically important because they drive organizational change and, as Rosabeth Moss-Kanter argues convincingly (Kanter et al., 1992), are at the core of successful change management. The essential ingredients of leadership, trust and ownership that are needed for effective change can only come about if these issues are recognized and properly addressed.

Why then are they given short shrift? Well, the simple answer is that they are difficult to deal with and there is no off-the-shelf solution. Tackling technical problems appears typically as a safer and more comfortable alternative to jumping into a fray of emotion-laden human relationships. Professionals such as managers, evaluators, auditors, etc. do not necessarily have the skills to deal effectively with people issues in this context. Also, the executive level of organizations often does not want to open up what it considers to be a Pandora's box, i.e. people issues are dealt with in the framework of 'labour relations'.

Yet evaluation can provide useful input on the 'people' issues as well. There is a considerable amount of know-how and literature on the 'people' side of 
evaluation process, e.g. participatory evaluation, empowerment evaluation, utilization-focused evaluation, fourth generation evaluation, etc.

Evaluators are in a position to, and should, draw more on the practice's wide span of knowledge, know-how and resources to address competently both the methodological and political aspects of evaluative enterprise, especially in the context of performance management initiatives.

\section{Lessons Learned}

The most important lesson for me is that performance management (and evaluative enterprise in general) cannot be forced on people. Attempting to impose it will likely lead to goal displacement, unreliable information and an increase in the risk that programme relevance will be diminished rather than augmented.

The success of a performance management process depends on the credibility it has with those who are involved. Credibility is needed for stakeholder buy-in, which, in turn, is necessary for the validation of the performance management process and its sustainability.

Because of the focus on all manner of results, which is the hallmark of current performance management initiatives in government, and the 'pendulum swings' that successive reforms impart on bureaucratic practice, it is easy to forget about process.

This leads me to another important lesson, which is that processes are as important as results. Among other things, this means valuing fully the quality of personal interaction. It also means ensuring that there are sufficient and appropriate resources to nurture process. The reality, however, is that as performance management initiatives are quite often undertaken because of resource constraints, it is not easy to convince the initiators that they should focus on, and invest in, process.

Another key lesson for me about dealing well with people is that some principles to guide the process and some 'rules of the game' should be laid out, discussed and agreed to with all participants.

For example, principles of fairness have always seemed especially important to me and equally valued by persons involved. I make a point of telling people at the outset of any audit I conduct that I follow two simple principles of fairness: you are accountable only for those things over which you have control, and you are not expected to do the impossible. In my experience, communicating these principles serves as a constructive starting point for collaborative enterprise.

Discussing and agreeing to some rules of the game is equally important, for example: making sure there is prior agreement on the interpretation of measures and the uses of performance information; instituting a mechanism to assess the performance management initiative or process itself (and to apply to it the expectations it applies to others); generating the active involvement and participation of stakeholders and ensuring transparency of process.

A final lesson learned is that independent input can be extremely helpful for developing an appropriate and credible performance management approach. The 


\section{Evaluation 5(2)}

auditor can play a very useful role by providing an external opinion on the soundness of the evaluative enterprise.

\section{Ideas to Work With}

One of the things I try to do in my practice is to expand the notion of performance from programme performance to organizational performance. In other words, to consider, for example, indicators that go beyond the linear resultsfocused family of measures to focus on aspects such as working environment, management direction, organizational responsiveness, safeguarding of human and physical assets, etc. Also, I attempt to foster an approach, a mindset, that uses indicators to elicit questions rather than to provide answers.

Another key idea is to develop performance information in order to cultivate learning. This approach simply recognizes that 'we don't know how to do it all', and we need to learn. This means that mistakes should be learning opportunities, and accepted as such.

Accountability should mean the ability to understand and explain what is going on; accountability for outcomes means the ability to demonstrate that 'you are keeping your eye on the outcome ball', and managing programme processes accordingly.

An idea that is central to sound and sustainable performance management is that knowledge, including assessment information, should be treated as a shared organizational resource rather than as an instrument of hierarchical power. To accept this view is to accept that some change in the way the organization functions is likely to be inevitable, while acknowledging that changes are accompanied by uncertainties.

According to David Garvin (1993), 'A learning organization is an organization skilled at creating, acquiring, and transferring knowledge, and at modifying its behaviour to reflect new knowledge and its insights'.

In his paper on building organizational capacity for performance measurement, Jim McDavid (1998) points out the interdependence between successful performance management systems and successful learning organizations. He goes on to expand on the five 'building blocks' Garvin suggests to create learning organizations, and provides us with a set of practical suggestions:

- Promote systematic problem solving by 'using a sequence of hypothesisgenerating, hypothesis-testing actions', and by relying on evidence rather than assumptions for decisions;

- Experiment changes to programmes in small and controlled ways by using pilot and demonstration projects;

- Performance data, and the tools to use them, should be widely disseminated within the organization;

- Learn from other organizations that do well to understand and apply their lessons learned;

- Institute mechanisms to transmit information and acquired knowledge effectively within the entire organization. 


\section{Concluding Remarks}

Whether evaluation or performance management, we are talking about change that involves people. The evaluator cannot alone bring about such change.

However, the evaluator can, and should, contribute know-how to help improve 'performance', recognizing that this contribution should include both methodological skills (in the 'technical' sense) and organizational change intervention skills (in the 'people' sense).

To achieve this, the focus should be on:

- A balanced attention to issues of process and results;

- Recognition of the limitations and difficulties and the need to learn;

- The treatment of information as a shared resource.

\section{Note}

This article was presented as a keynote address at the annual conference of the United Kingdom Evaluation Society, December 1998, London.

\section{References}

English, J. and E. Lindquist (1998) Performance Management: Linking Results to Public Debate. Ottawa.

European Commission (1997) Evaluating EU Expenditure Programmes: A Guide to Ex Post and Intermediate Evaluation. Luxembourg: European Commission, January.

Garvin, D. A. (1993) 'Building a Learning Organization', Harvard Business Review XX, July-August.

Kanter, R. M., B. A. Stein and T. D. Jick (1992) The Challenge of Organizational Change: How Companies Experience It and Leaders Guide It. Toronto: Maxwell Macmillan Canada.

McDavid, J. C. (1998) Building Organizational Capacity for Performance Measurement: Mile Posts, Toll Booths, and Construction Zones on the Yellow Brick Road. Victoria: Local Government Institute.

Perrin, B. (1999) 'Effective Use and Misuse of Performance Measurement', American Journal of Evaluation 20(1).

Treasury Board of Canada (1998) Managing for Results. Ottawa: Treasury Board of Canada Secretariat.

United States General Accounting Office (1998) Performance Measurement and Evaluation: Definitions and Relationships. Washington, DC: USGAO.

IAN C. DAVIES is Advisor to the European Court of Auditors on performance audit, evaluation and sound financial management. Mr Davies is also a member of the External Advisory Panel to the President of the Treasury Board of Canada on results measurement and accountability. He has advised the Directorate General for Financial Control of the European Commission on evaluation, performance audit and the modernization of controllership. Mr Davies has worked for the Auditor General of British Columbia (Canada) and the governments of Canada and the province of Québec. Please address correspondence to: European Court of Auditors, 12, rue Alcide De Gasperi, L-1615 Luxembourg. [email: ian.davies@eca.eu.int] 